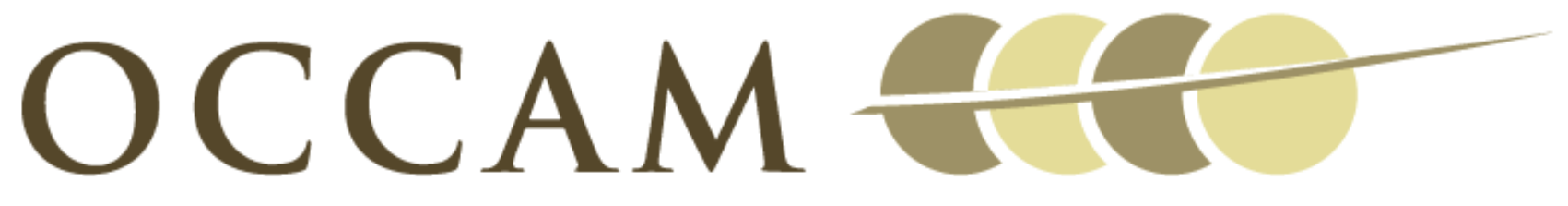

OXFORD CENTRE FOR COLLABORATIVE APPLIED MATHEMATICS

Report Number 10/52

Order parameters in the Landau-de Gennes theory - the static and dynamic scenarios

by

Apala Majumdar

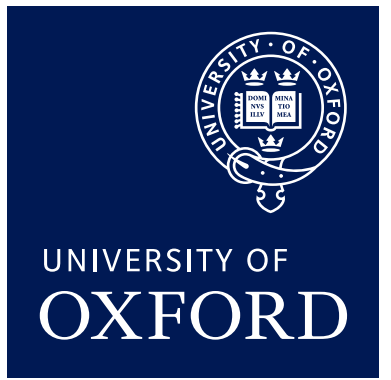

Oxford Centre for Collaborative Applied Mathematics Mathematical Institute 24 - 29 St Giles'

Oxford

OX1 3LB

England 



\title{
Order parameters in the Landau-de Gennes theory - the static and dynamic scenarios
}

\author{
Apala Majumdar*
}

July 8, 2010

\begin{abstract}
We obtain quantitative estimates for the scalar order parameters of liquid crystal configurations in three-dimensional geometries, within the Landau-de Gennes framework. We consider both static equilibria and non-equilibrium dynamics and we include external fields and surface anchoring energies into our formulation. Using maximum principletype arguments, we obtain explicit bounds for the corresponding scalar order parameters in both static and dynamic situations; these bounds are given in terms of the material-dependent thermotropic coefficients, electric field strength and surface anchoring coefficients. These bounds provide estimates for the degree of orientational ordering, quantify the competing effects of the different energetic contributions and can be used to test the accuracy of numerical simulations.
\end{abstract}

Keywords: Nematic liquid crystals, order parameters, electric fields, surface anchoring, Landau-de Gennes theory

AMS Classification: 35Qxx, 35Jxx, 35Bxx

\section{Introduction}

Recent years have seen a burgeoning demand for liquid crystals as softmatter based devices in the display industry and in nanotechnology [19]. The simplest liquid crystal phase is the nematic phase, where the constituent rod-like molecules have no translational order but exhibit a certain degree of long-range orientational ordering. The resulting anisotropic physical and optical properties make liquid crystals suitable for technological and biological applications. There is now a growing need to systematically model liquid crystals in prototype device geometries and transfer this modelling to the analysis of numerical simulations and interpretation of physical experiments.

\footnotetext{
${ }^{*}$ Mathematical Institute, University of Oxford, 24-29 St.Giles, Oxford, OX1 3LB (majumdar@maths.ox.ac.uk).
} 
In this paper, we continue the work initiated in [14] and consider various physically relevant generalizations. In [14], we rigorously compare two widely-used and widely-accepted approaches to the mathematical modelling of nematic liquid crystals - mean-field approaches $[6,18]$ and the continuum Landau-de Gennes theory $[6,16,12]$. In both theoretical frameworks, the state of a nematic liquid crystal is described by a symmetric, traceless $3 \times 3$ matrix - the $\mathbf{Q}$-tensor order parameter. The $\mathbf{Q}$-tensor order parameter contains information about the degree of orientational ordering within its eigenvalues and the directions of preferred alignment within its eigenvectors [16]. The degree of orientational ordering is often conveniently given in terms of scalar order parameters, which are defined to be appropriate linear combinations of the eigenvalues. Mean-field approaches define the Q-tensor order parameter in terms of the second moment of the probability distribution function $\psi$ for the molecular orientations [6]. This probabilistic definition of the $\mathbf{Q}$-tensor requires the corresponding eigenvalues, $\lambda_{i}(\mathbf{Q})$, to be bounded from both above and below i.e. $\lambda_{i}(\mathbf{Q}) \in\left[-\frac{1}{3}, \frac{2}{3}\right][14,8,9]$. On the other hand, the $\mathbf{Q}$-tensor is defined in terms of anisotropic macroscopic quantities such as the magnetic susceptibility or dielectric anisotropy in the Landau-de Gennes framework. This definition is independent of $\psi$ and there are no a priori constraints on the eigenvalues i.e. $\lambda_{i}(\mathbf{Q})$ and hence, the scalar order parameters can in principle be arbitrarily large [6].

In [14], we study static equilibrium liquid crystal configurations in threedimensional geometries subject to Dirichlet boundary conditions, within the continuum Landau-de Gennes framework. These static equilibria are given by either global or local minimizers of the corresponding Landau-de Gennes energy functional, which is a nonlinear integral functional of the $\mathbf{Q}$-tensor and its spatial derivatives. We consider both spatially homogeneous and spatially inhomogeneous cases in [14], for all temperature regimes. The global energy minimizers can be explicitly computed in the spatially homogeneous cases and in the spatially inhomogeneous cases, we compute an explicit upper bound for the norm of a global energy minimizer in terms of the temperature, material-dependent thermotropic constants and the boundary condition. We use these bounds to compare the Landau-de Gennes theoretical predictions with the mean-field theoretical predictions. In [14], we explicitly delineate the parameter regimes of agreement and disagreement between Landau-de Gennes theory and mean-field theory, for both homogeneous and inhomogeneous cases. We find that the Landau-de Gennes theory can predict large values for the equilibrium scalar order parameters, larger than unity, in the low-temperature regime and this low-temperature regime can be explicitly specified in terms of material-dependent constants. For the commonly used liquid crystal material MBBA, the Landau-de Gennes predictions fail to be physically realistic when we move to temperatures just $2^{\circ} \mathrm{C}$ below the nematic-isotropic transition temperature.

In this paper, we consider various generalizations of the problem studied 
in [14]. In [14], we work in a simple framework i.e. in the absence of external fields and with Dirichlet boundary conditions (known as strong anchoring in the liquid crystal literature) in static situations. In Section 2 of this paper, we recall the main results in [14] and generalize these results to a Landau-de Gennes energy functional with an electric-field energy and a physically realistic surface energy density. This surface energy density promotes alignment either parallel or perpendicular to a given direction on the boundary, depending on the values of the surface anchoring coefficients [11]. We compute the governing equations for the static equilibria in both cases and use elegant maximum-principle type arguments to compute bounds for the norm of static equilibria in terms of the temperature, thermotropic constants, electric field strength and surface anchoring coefficents. This result is relevant to the interpretation of physical experiments which often take place in the presence of external fields and the experimental set-up can almost never realize the idealized framework of Dirichlet boundary conditions. In Section 3, we consider the non-equilibrium dynamics of these confined liquid crystal systems under gradient flow of the associated Landau-de Gennes energy functional and we compute bounds for the norm of the evolving liquid crystal configuration in space and time. This result relies on maximumprinciple type arguments for parabolic partial differential equations. The results in Sections 2 and 3 are restricted to the one-constant elastic energy density, which is a widely-used form of the elastic energy density, especially for analytic purposes. In Section 4, we consider a very general form of the elastic energy density with three elastic constants [5]. We explicitly compute the governing equations in this case and outline the main mathematical difficulties here. In Section 5, we present our conclusions and directions for future research.

\section{Time-independent problems}

In this section, we focus on the qualitative properties of stable liquid crystal configurations in static situations, in the Landau-de Gennes framework. The macroscopic order parameter is a symmetric, traceless $3 \times 3$ matrix - the $\mathbf{Q}$ tensor order parameter. Let $\bar{S} \subset \mathbb{M}^{3 \times 3}$ denote the space of symmetric, traceless $3 \times 3$ matrices i.e.

$$
\bar{S} \stackrel{\text { def }}{=}\left\{\mathbf{Q} \in \mathbb{M}^{3 \times 3} ; \mathbf{Q}_{i j}=\mathbf{Q}_{j i}, \mathbf{Q}_{i i}=0\right\}
$$

where we have used the Einstein summation convention; the Einstein convention will be used in the rest of the paper. An arbitrary $\mathbf{Q} \in \bar{S}$ can be written as

$$
\mathbf{Q}=S\left(\mathbf{n} \otimes \mathbf{n}-\frac{1}{3} \mathbf{I}\right)+R(\mathbf{m} \otimes \mathbf{m}-\mathbf{p} \otimes \mathbf{p})
$$


where $\mathbf{n}, \mathbf{m}, \mathbf{p}$ are orthonormal eigenvectors of $\mathbf{Q}$ and $S, R$ are scalar order parameters constrained by the inequalities $0 \leq R \leq \frac{S}{3}$ or $\frac{S}{3} \leq R \leq 0$ $[16,13]$. We refer to $(S, R)$ as scalar order parameters in the rest of the paper. Nematic liquid crystals are said to be in the (a) biaxial phase when the corresponding $\mathbf{Q}$-tensor has three distinct eigenvalues, (b) uniaxial phase when $\mathbf{Q}$ has a pair of equal non-zero eigenvalues and (c) isotropic phase when $\mathbf{Q}$ has three equal eigenvalues or equivalently when $\mathbf{Q}=0$. The corresponding matrix-norm is defined to be

$$
|\mathbf{Q}| \stackrel{\text { def }}{=} \sqrt{\operatorname{tr} \mathbf{Q}^{2}}=\sqrt{\mathbf{Q}_{i j} \mathbf{Q}_{i j}} \quad i, j=1 \ldots 3 .
$$

Equivalently, the matrix norm is related to $(S, R)$ by

$$
|\mathbf{Q}|^{2}=\frac{2}{3} S^{2}+2 R^{2}
$$

and therefore, bounds for $|\mathbf{Q}|$ directly translate to bounds for the corresponding scalar order parameters.

We take our domain $\Omega$ to be a three-dimensional bounded, connected and simply-connected set with smooth boundary, $\partial \Omega$. We work with the following form of the Landau-de Gennes energy functional:

$$
I_{L G}[\mathbf{Q}]=\int_{\Omega} \frac{L}{2}|\nabla \mathbf{Q}|^{2}+f_{B}(\mathbf{Q})+F(\mathbf{Q}, \mathbf{E}) d V+\int_{\partial \Omega} G(\mathbf{Q}) d S
$$

where $|\nabla \mathbf{Q}|^{2}$ is the one-constant elastic energy density, $L>0$ is a materialdependent elastic constant, $f_{B}$ is a bulk energy density that dictates the preferred phase of the liquid crystal - isotropic, uniaxial or biaxial, $F$ is an external field energy density where $\mathbf{E}$ represents the external field and $G$ is a surface energy density. Specific forms for $F$ and $G$ will be specified later. We work with the simplest form of the bulk energy density, $f_{B}$, in (3) that allows for a first-order nematic-isotropic phase transition [16]. We take $f_{B}$ to be a quartic polynomial in the $\mathbf{Q}$-tensor invariants as shown below -

$$
f_{B}(\mathbf{Q})=\frac{a^{2}}{2} \operatorname{tr}\left(\mathbf{Q}^{2}\right)-\frac{b^{2}}{3} \operatorname{tr}\left(\mathbf{Q}^{3}\right)+\frac{c^{2}}{4}\left(\operatorname{tr}\left(\mathbf{Q}^{2}\right)\right)^{2}+C\left(a^{2}, b^{2}, c^{2}\right)
$$

where $a^{2}=\alpha\left(T-T^{*}\right), \alpha$ is a characteristic material-dependent positive constant and $T^{*}$ is a characteristic temperature, $b^{2}, c^{2} \in \mathbb{R}^{+}$are materialdependent positive constants and $C\left(a^{2}, b^{2}, c^{2}\right)$ is a positive constant that ensures $f_{B}(\mathbf{Q}) \geq 0$ for all $\mathbf{Q} \in \bar{S}$. We note that $C\left(a^{2}, b^{2}, c^{2}\right)$ plays no role in energy minimization, in either spatially homogeneous or inhomogeneous cases. The stationary points of $f_{B}$ correspond to either uniaxial or isotropic Q-tensors $[1,14]$. There are three characteristic temperature regimes for $f_{B}$ in (4): i) $a^{2}=0$, below which the isotropic state $\mathbf{Q}=0$ loses its stability and $f_{B}$ attains its minimum on the set of uniaxial $\mathbf{Q}$-tensors given by

$$
\mathbf{Q}_{\min }=\left\{\mathbf{Q} \in \bar{S} ; \mathbf{Q}=s_{+}\left(\mathbf{n} \otimes \mathbf{n}-\frac{1}{3} \mathbf{I}\right)\right\}
$$


and $\mathbf{n} \in \mathbb{S}^{2}$ and

$$
s_{+}=\frac{b^{2}+\sqrt{b^{4}-24 a^{2} c^{2}}}{4 c^{2}},
$$

(ii) the nematic-isotropic transition temperature, $a^{2}=\frac{b^{4}}{27 c^{2}}$, for which $f_{B}\left(\mathbf{Q}_{\min }\right)=$ $f_{B}(\mathbf{0})$ and (iii) $a^{2}=\frac{b^{4}}{24 c^{2}}$ above which isotropic state is the unique stationary point of $f_{B}$ in (4).

In what follows, we first recall a result from [14, 13] where we do not account for $F$ and $G$ in (3) i.e.work with Dirichlet boundary conditions in the absence of external fields. This result is then generalized to account for an external electric field in Proposition 2 and to include a general surface energy density in Proposition 4.

Proposition 1. [14, 13] Consider the Landau-de Gennes energy functional

$$
I_{L G}[\mathbf{Q}]=\int_{\Omega} \frac{L}{2}|\nabla \mathbf{Q}|^{2}+f_{B}(\mathbf{Q}) d V
$$

where $f_{B}$ is defined in (4), in the admissible space

$$
\mathcal{A}=\left\{\mathbf{Q} \in W^{1,2}(\Omega ; \bar{S}) ; \mathbf{Q}=\mathbf{Q}_{b} \text { on } \partial \Omega\right\}
$$

where

$$
\left|\mathbf{Q}_{b}(\mathbf{x})\right| \leq \frac{1}{\sqrt{6}} s_{+} \quad \mathbf{x} \in \partial \Omega
$$

where $s_{+}$has been defined in (6). (Recall that $W^{1,2}(\Omega ; \bar{S})$ is the Sobolev space of square-integrable $\mathbf{Q}$-tensors with square-integrable first derivatives [7]. The corresponding $W^{1,2}$-norm is defined to be $\|\mathbf{Q}\|_{W^{1,2}(\Omega)}=\left(\int_{\Omega}|\mathbf{Q}|^{2}+|\nabla \mathbf{Q}|^{2} d x\right)^{1 / 2}$.) The corresponding Euler-Lagrange equations are given by the following system of coupled elliptic partial differential equations :

$$
L \Delta \mathbf{Q}_{i j}=a^{2} \mathbf{Q}_{i j}-b^{2}\left(\mathbf{Q}_{i k} \mathbf{Q}_{k j}-\frac{\delta_{i j}}{3} \operatorname{tr}\left(\mathbf{Q}^{2}\right)\right)+c^{2} \mathbf{Q}_{i j} \operatorname{tr}\left(\mathbf{Q}^{2}\right) \quad i, j=1,2,3 .
$$

Let $\mathbf{Q}$ be an arbitrary solution of the system (9). Then $\mathbf{Q}$ is necessarily a classical solution of (9) and satisfies the following global upper bound on $\bar{\Omega}$ :

$$
|\mathbf{Q}(\mathbf{x})| \leq \sqrt{\frac{2}{3}} s_{+} \quad \mathbf{x} \in \bar{\Omega} .
$$

Comment: The bound (10) is independent of $L$ and is clearly satisfied on $\partial \Omega$, by virtue of the hypothesis on $\mathbf{Q}_{b}$. In the limit $L \rightarrow 0^{+}$, global minimizers of (7) approach the upper bound (10) arbitrarily closely, in the interior of $\Omega$, away from topological singularities [13]. We note that the $L \rightarrow 0$ limit is equivalent to the limit $\bar{L}=\frac{27 L c^{2}}{b^{4} R^{2}} \rightarrow 0$, which is referred to as the vanishing core limit and is relevant for macroscopic domains with characteristic size $R>>\sqrt{\frac{27 L c^{2}}{b^{4}}}[17,15]$. 
Proof: The proof proceeds by contradiction. We first consider the low temperature regime $a^{2} \leq \frac{b^{4}}{24 c^{2}}$. Let $\mathbf{Q}$ be an arbitrary classical solution of (9) for a fixed $L>0$. We assume that there exists a $\mathbf{x}^{*} \in \Omega$ where $|\mathbf{Q}|$ attains its maximum and $|\mathbf{Q}|\left(\mathbf{x}^{*}\right)>\sqrt{\frac{2}{3}} s_{+}$. Therefore, the function $|\mathbf{Q}|^{2}: \bar{\Omega} \rightarrow \mathbb{R}$ must necessarily attain its maximum at $\mathbf{x}^{*}$ and we have

$$
\Delta\left(\frac{1}{2}|\mathbf{Q}|^{2}\right)\left(\mathbf{x}^{*}\right) \leq 0
$$

by definition of an interior maximum point [7].

We multiply both sides of (9) by $\mathbf{Q}_{i j}$ to obtain

$$
L \Delta\left(\frac{1}{2}|\mathbf{Q}|^{2}\right)=a^{2} \operatorname{tr} \mathbf{Q}^{2}-b^{2} \operatorname{tr} \mathbf{Q}^{3}+c^{2}\left(\operatorname{tr} \mathbf{Q}^{2}\right)^{2}+L|\nabla \mathbf{Q}|^{2}
$$

One can directly check that

$$
a^{2} \operatorname{tr} \mathbf{Q}^{2}-b^{2} \operatorname{tr} \mathbf{Q}^{3}+c^{2}\left(\operatorname{tr} \mathbf{Q}^{2}\right)^{2} \geq f(|\mathbf{Q}|)
$$

where

$$
f(|\mathbf{Q}|)=c^{2}|\mathbf{Q}|^{2}\left(|\mathbf{Q}|-\sqrt{\frac{2}{3}} s_{+}\right)\left(|\mathbf{Q}|-Q_{-}\right),
$$

$Q_{-}=\sqrt{\frac{2}{3}}\left(\frac{b^{2}-\sqrt{b^{4}-24 a^{2} c^{2}}}{4 c^{2}}\right)$ and we have used the inequality $\operatorname{tr} \mathbf{Q}^{3} \leq \frac{|\mathbf{Q}|^{3}}{\sqrt{6}}$ from $[14,13]$. It immediately follows from (14) that if $|\mathbf{Q}|\left(\mathbf{x}^{*}\right)>\sqrt{\frac{2}{3}} s_{+}$, then $f(|\mathbf{Q}|)\left(\mathbf{x}^{*}\right)>0$ and from (12) and (13), $\Delta\left(\frac{1}{2}|\mathbf{Q}|^{2}\right)\left(\mathbf{x}^{*}\right)>0$, contradicting the hypothesis (11). Therefore,

$$
|\mathbf{Q}|\left(\mathbf{x}^{*}\right) \leq \sqrt{\frac{2}{3}} s_{+}
$$

and Proposition 1 now follows.

In the high-temperature regime, $a^{2}>\frac{b^{4}}{24 c^{2}}, s_{+}$and $Q_{-}$are not defined and $\Delta\left(\frac{1}{2}|\mathbf{Q}|^{2}\right)>0$ everywhere in $\Omega$ and $|\mathbf{Q}|$ attains its maximum on $\partial \Omega$.

In Proposition 2, we consider the interaction of an externally applied electric field with a nematic liquid crystal. In principle, the electric field within the liquid crystal cell is unknown and should be computed using Maxwell's equations $[16,12]$. However, for simplicity, researchers often take the applied electric field, $\mathbf{E}=\left(E_{1}, E_{2}, E_{3}\right)$, to be known and constant throughout the cell and we adopt this approach here. Secondly, we neglect the effects of flexoelectricity or spontaneous polarisation [16] and take the electric energy density to be

$$
F(\mathbf{Q}, \mathbf{E})=-\frac{\epsilon_{0}}{2}\left(\triangle \epsilon \mathbf{E}_{i} \mathbf{Q}_{i j} \mathbf{E}_{j}+\bar{\epsilon}|\mathbf{E}|^{2}\right) \quad i, j=1 \ldots 3
$$


where $\epsilon_{0}$ is the permittivity of free space, $\triangle \epsilon=\left(\epsilon_{\|}-\epsilon_{\perp}\right) / S_{\text {exp }}$ is the scaled dielectric anisotropy $\left(\epsilon_{\|}\left(\epsilon_{\perp}\right)\right.$ is the dielectric permittivity parallel to $\mathbf{E}$ (perpendicular to E) respectively and $S_{\text {exp }}$ is an experimentally measured constant) and $\bar{\epsilon}=\left(\epsilon_{\|}+2 \epsilon_{\perp}\right) / 3$ is an average permittivity [16].

Proposition 2. Consider the Landau-de Gennes energy functional

$$
I_{L G}[\mathbf{Q}]=\int_{\Omega} \frac{L}{2}|\nabla \mathbf{Q}|^{2}+f_{B}(\mathbf{Q})+F(\mathbf{Q}, \mathbf{E}) d V
$$

where $f_{B}$ is defined in (4), $F(\mathbf{Q}, \mathbf{E})$ is defined in (15) and the admissible space is defined to be

$$
\mathcal{A}=\left\{\mathbf{Q} \in W^{1,2}(\Omega ; \bar{S}) ; \mathbf{Q}=\mathbf{Q}_{b} \text { on } \partial \Omega\right\} .
$$

The fixed boundary condition $\mathbf{Q}_{b}$ satisfies the inequality

$$
\left|\mathbf{Q}_{b}\right|(\mathbf{x}) \leq \frac{1}{2} F_{3} \quad \mathbf{x} \in \partial \Omega
$$

where $F_{3}$ is the largest positive zero of the cubic polynomial

$$
|\mathbf{Q}|^{3}-\frac{b^{2}}{\sqrt{6} c^{2}}|\mathbf{Q}|^{2}+\frac{a^{2}}{c^{2}}|\mathbf{Q}|-\epsilon_{0} \triangle \epsilon \frac{|\mathbf{E}|^{2}}{\sqrt{6} c^{2}}=0 .
$$

The corresponding Euler-Lagrange equations are given by

$L \Delta \mathbf{Q}_{i j}=a^{2} \mathbf{Q}_{i j}-b^{2}\left(\mathbf{Q}_{i k} \mathbf{Q}_{k j}-\frac{\delta_{i j}}{3} \operatorname{tr}\left(\mathbf{Q}^{2}\right)\right)+c^{2} \mathbf{Q}_{i j} \operatorname{tr}\left(\mathbf{Q}^{2}\right)-\frac{1}{2} \epsilon_{0} \Delta \epsilon\left(\mathbf{E}_{i} \mathbf{E}_{j}-\frac{|\mathbf{E}|^{2}}{3} \delta_{i j}\right) \quad i, j=1,2,3$.

Let $\mathbf{Q}$ be an arbitrary solution of (20). Then $\mathbf{Q}$ is necessarily a classical solution of (20) and

$$
|\mathbf{Q}|(\mathbf{x}) \leq F_{3}\left(a^{2}, b^{2}, c^{2}, \triangle \epsilon,|\mathbf{E}|^{2}\right) \quad \mathbf{x} \in \bar{\Omega} .
$$

Proof: Consider the Landau-de Gennes energy functional (16). It is straightforward to verify that the corresponding Euler-Lagrange equations are indeed given by (20), where the terms $b^{2} \frac{\delta_{i j}}{3} \operatorname{tr}\left(\mathbf{Q}^{2}\right)$ and $\frac{1}{2} \epsilon_{0} \triangle \epsilon \frac{|\mathbf{E}|^{2}}{3} \delta_{i j}$ are Lagrange multipliers accounting for the tracelessness constraint. We assume that the function $|\mathbf{Q}|: \Omega \rightarrow \mathbb{R}^{+}$attains its maximum at an interior point $\mathbf{x}^{*} \in \Omega$ and $|\mathbf{Q}|\left(\mathbf{x}^{*}\right)>F_{3}$ where $F_{3}$ has been defined in (19). Then

$$
\Delta\left(\frac{1}{2}|\mathbf{Q}|^{2}\right)\left(\mathbf{x}^{*}\right) \leq 0
$$

by definition of an interior maximum point [7].

As in Proposition 1, we multiply both sides of (20) by $\mathbf{Q}_{i j}$ to obtain the following:

$$
L \Delta\left(\frac{1}{2}|\mathbf{Q}|^{2}\right)=a^{2} \operatorname{tr} \mathbf{Q}^{2}-b^{2} \operatorname{tr} \mathbf{Q}^{3}+c^{2}\left(\operatorname{tr} \mathbf{Q}^{2}\right)^{2}-\frac{1}{2} \epsilon_{0} \triangle \epsilon \mathbf{E}_{i} \mathbf{Q}_{i j} \mathbf{E}_{j}+L|\nabla \mathbf{Q}|^{2} .
$$


Next, we claim that

$$
\left|\mathbf{E}_{i} \mathbf{Q}_{i j} \mathbf{E}_{j}\right| \leq \sqrt{\frac{2}{3}}|\mathbf{Q}||\mathbf{E}|^{2} \quad i, j=1 \ldots 3 .
$$

Using the representation (1), we obtain the following explicit expression for $\left(\mathbf{E}_{i} \mathbf{Q}_{i j} \mathbf{E}_{j}\right)^{2}$ : -

$$
\begin{aligned}
& \left(\mathbf{E}_{i} \mathbf{Q}_{i j} \mathbf{E}_{j}\right)^{2}=S^{2}\left[(\mathbf{n} \cdot \mathbf{E})^{4}-\frac{2}{3}(\mathbf{n} \cdot \mathbf{E})^{2}|\mathbf{E}|^{2}+\frac{1}{9}|\mathbf{E}|^{4}\right]+ \\
& \quad+\frac{2 S R}{3}\left((\mathbf{n} \cdot \mathbf{E})^{2}-\frac{|\mathbf{E}|^{2}}{3}\right)\left[(\mathbf{m} \cdot \mathbf{E})^{2}-(\mathbf{p} \cdot \mathbf{E})^{2}\right]+R^{2}\left[(\mathbf{m} \cdot \mathbf{E})^{2}-\left(\mathbf{p} \cdot \mathbf{E}\left\langle 22^{2}\right)^{2}\right.\right.
\end{aligned}
$$

where either $0 \leq R \leq \frac{S}{3}$ or $\frac{S}{3} \leq R \leq 0$. At this point, it suffices to note that

$$
(\mathbf{n} \cdot \mathbf{E})^{2}+(\mathbf{m} \cdot \mathbf{E})^{2}+(\mathbf{p} \cdot \mathbf{E})^{2}=|\mathbf{E}|^{2}
$$

since $\mathbf{n}, \mathbf{m}, \mathbf{p}$ form a triad of orthonormal eigenvectors of $\mathbf{Q}$, at each point $\mathbf{x} \in \Omega$. Then

$$
(\mathbf{m} \cdot \mathbf{E})^{2}-(\mathbf{p} \cdot \mathbf{E})^{2} \leq|\mathbf{E}|^{2}-(\mathbf{n} \cdot \mathbf{E})^{2} \leq|\mathbf{E}|^{2} .
$$

Using the above in (25), we obtain the following upper bound for $\left(\mathbf{E}_{i} \mathbf{Q}_{i j} \mathbf{E}_{j}\right)^{2}$ as shown below: -

$$
\begin{aligned}
& \left(\mathbf{E}_{i} \mathbf{Q}_{i j} \mathbf{E}_{j}\right)^{2} \leq S^{2}\left[(\mathbf{n} \cdot \mathbf{E})^{4}-\frac{2}{3}(\mathbf{n} \cdot \mathbf{E})^{2}|\mathbf{E}|^{2}+\frac{1}{9}|\mathbf{E}|^{4}\right]+ \\
& +\frac{2 S R}{3}\left((\mathbf{n} \cdot \mathbf{E})^{2}+\frac{|\mathbf{E}|^{2}}{3}\right)\left(|\mathbf{E}|^{2}-(\mathbf{n} \cdot \mathbf{E})^{2}\right)+|\mathbf{E}|^{4} R^{2}
\end{aligned}
$$

It is straightforward to check that the function

$$
h\left((\mathbf{n} \cdot \mathbf{E})^{2}\right)=S^{2}\left[(\mathbf{n} \cdot \mathbf{E})^{4}-\frac{2}{3}(\mathbf{n} \cdot \mathbf{E})^{2}|\mathbf{E}|^{2}+\frac{1}{9}|\mathbf{E}|^{4}\right]+\frac{2 S R}{3}\left((\mathbf{n} \cdot \mathbf{E})^{2}+\frac{|\mathbf{E}|^{2}}{3}\right)\left(|\mathbf{E}|^{2}-(\mathbf{n} \cdot \mathbf{E})^{2}\right)
$$

attains its maximum over the range $(\mathbf{n} \cdot \mathbf{E})^{2} \in\left[0,|\mathbf{E}|^{2}\right]$, at the end-point $(\mathbf{n} \cdot \mathbf{E})^{2}=|\mathbf{E}|^{2}$ and

$$
h\left((\mathbf{n} \cdot \mathbf{E})^{2}\right) \leq \frac{4 S^{2}}{9}|\mathbf{E}|^{4} .
$$

Therefore,

$$
\left(\mathbf{E}_{i} \mathbf{Q}_{i j} \mathbf{E}_{j}\right)^{2} \leq \frac{2}{3}|\mathbf{E}|^{4}\left(\frac{2 S^{2}}{3}+2 R^{2}\right) .
$$

Finally, we note from (1) that

$$
|\mathbf{Q}|^{2}=\left(\frac{2 S^{2}}{3}+2 R^{2}\right)
$$


and combining the above with (28), the claim (24) follows.

We substitute (24) into (23) to obtain the following lower bound: -$$
L \Delta\left(\frac{1}{2}|\mathbf{Q}|^{2}\right) \geq a^{2} \operatorname{tr} \mathbf{Q}^{2}-b^{2} \operatorname{tr} \mathbf{Q}^{3}+c^{2}\left(\operatorname{tr} \mathbf{Q}^{2}\right)^{2}-\frac{\epsilon_{0} \triangle \epsilon}{\sqrt{6}}|\mathbf{E}|^{2}|\mathbf{Q}|
$$$$
\geq c^{2}|\mathbf{Q}|\left(|\mathbf{Q}|^{3}-\frac{b^{2}}{\sqrt{6} c^{2}}|\mathbf{Q}|^{2}+\frac{a^{2}}{c^{2}}|\mathbf{Q}|-\frac{\epsilon_{0} \Delta \epsilon}{\sqrt{6} c^{2}}|\mathbf{E}|^{2}\right)
$$

$$
H_{e}(|\mathbf{Q}|)=\left(|\mathbf{Q}|^{3}-\frac{b^{2}}{\sqrt{6} c^{2}}|\mathbf{Q}|^{2}+\frac{a^{2}}{c^{2}}|\mathbf{Q}|-\frac{\epsilon_{0} \triangle \epsilon}{\sqrt{6} c^{2}}|\mathbf{E}|^{2}\right)
$$

is a cubic polynomial in $|\mathbf{Q}|, H_{e}(|\mathbf{Q}|) \rightarrow \infty$ as $|\mathbf{Q}| \rightarrow \infty$ (since $\left.c^{2}>0\right), H_{e}$ has three zeros:-

$$
F_{1} \leq F_{2} \leq F_{3}
$$

and $H_{e}>0$ for all $|\mathbf{Q}|>F_{3}$.

Therefore, if $|\mathbf{Q}|\left(\mathbf{x}^{*}\right)>F_{3}$, then

$$
\Delta\left(\frac{1}{2}|\mathbf{Q}|^{2}\right)\left(\mathbf{x}^{*}\right)>0
$$

from (29), contradicting the hypothesis (22). Therefore,

$$
|\mathbf{Q}|(\mathbf{x}) \leq F_{3} \quad \mathbf{x} \in \bar{\Omega}
$$

where $F_{3}$ is the largest positive real zero of the cubic polynomial (19) and Proposition 2 follows.

Comment: Proposition 2 would also hold if $\mathbf{E}$ were spatially varying in $\Omega$ i.e. if we had an inhomogeneous electric field in the material.

One would add a flexoelectric term to the energy density as shown below:

$$
F_{\text {flexo }}(\mathbf{Q}, \mathbf{E})=-\frac{\epsilon_{0}}{2}\left(\triangle \epsilon \mathbf{E}_{i} \mathbf{Q}_{i j} \mathbf{E}_{j}+\bar{\epsilon}|\mathbf{E}|^{2}\right)-\bar{e} \mathbf{Q}_{i j, j} \mathbf{E}_{i} \quad i, j=1 \ldots 3
$$

where $\bar{e}$ is a material-dependent constant [16]. The corresponding EulerLagrange equations for the energy functional

$$
I_{L G}[\mathbf{Q}]=\int_{\Omega} \frac{L}{2}|\nabla \mathbf{Q}|^{2}+f_{B}(\mathbf{Q})+F_{\text {flexo }}(\mathbf{Q}, \mathbf{E}) d V
$$

are identical to (20) and hence, the upper bound for the corresponding solution norms in (21) is unchanged.

The next step is to relax the assumption of fixed dirichlet boundary conditions and consider a general surface energy density, such as the one proposed in [11].

$$
G(\mathbf{Q})=W_{11} \mathbf{e}_{i} \mathbf{Q}_{i j} \mathbf{e}_{j}+W_{21} \operatorname{tr} \mathbf{Q}^{2}+W_{22}\left(\mathbf{e}_{i} \mathbf{Q}_{i j} \mathbf{e}_{j}\right)^{2}+W_{23}\left(\mathbf{Q}_{i j} \mathbf{e}_{j}\right)^{2}
$$


where $\mathbf{e}$ is a prescribed unit-vector and $W_{11}, W_{21}, W_{22}, W_{23}$ are surface anchoring coefficients. There are two immediate questions - (a) what constraints are needed on the surface anchoring coefficients to ensure that $G(\mathbf{Q})$ is bounded from below for all $\mathbf{Q} \in \bar{S}$ and (b) can we compute the corresponding minimizers of $G$ in (30)?

Proposition 3. The surface energy density in (30) is bounded from below

$$
G(\mathbf{Q}) \geq-\frac{W_{11}^{2}}{4\left(\frac{3}{2} W_{21}+W_{22}+W_{23}\right)}
$$

for all $\mathbf{Q} \in \bar{S}$ if the surface anchoring coefficients satisfy the inequalities:

$$
W_{21}>0 ; 2 W_{21}+W_{23}>0 ; \frac{3}{2} W_{21}+W_{22}+W_{23}>0 .
$$

Proof: The constraints (32) are given in [11]. We give a simple alternative proof of the well-posedness of the surface energy density under these constraints, for the reader's interest.

Consider the symmetric, traceless $3 \times 3$ matrix

$$
E_{i j}^{0}=\left(\mathbf{e}_{i} \mathbf{e}_{j}-\frac{1}{3} \delta_{i j}\right)
$$

where $\mathbf{e}_{i}$ denotes the $i$-th component of $\mathbf{e}$ and $i, j=1 \ldots 3$. Then we can construct a set of five mutually orthogonal matrices $\left(E^{0}, E^{1}, E^{2}, E^{3}, E^{4}\right) \in$ $\bar{S}$ with $E^{k_{1}} \cdot E^{k_{2}}=E_{i j}^{k_{1}} E_{i j}^{k_{2}}=0$ for $k_{1} \neq k_{2}, i, j=1 \ldots 3$, such that $\left(E^{0}, E^{1}, E^{2}, E^{3}, E^{4}\right)$ form a basis for $\bar{S}$ (see [12] for similar arguments). More precisely, let $\mathbf{e}, \mathbf{f}, \mathbf{g}$ form an orthonormal basis for $\mathbb{R}^{3}$. One can check that $E^{0}$ as defined above and

$$
\begin{aligned}
& E^{1}=\left(\mathbf{e}_{i} \mathbf{f}_{j}-\mathbf{e}_{j} \mathbf{f}_{i}\right) \\
& E^{2}=\left(\mathbf{e}_{i} \mathbf{g}_{j}-\mathbf{g}_{i} \mathbf{e}_{j}\right) \\
& E^{3}=\left(\mathbf{f}_{i} \mathbf{g}_{j}-\mathbf{g}_{i} \mathbf{f}_{j}\right) \\
& E^{4}=\left(\mathbf{f}_{i} \mathbf{f}_{j}-\mathbf{g}_{i} \mathbf{g}_{j}\right)
\end{aligned}
$$

form an orthogonal basis for $\bar{S}$. Then any $\mathbf{Q} \in \bar{S}$ can be written as

$$
\mathbf{Q}_{i j}=\lambda E_{i j}^{0}+\sum_{k=1}^{4} \delta_{k} E_{i j}^{k}
$$

where $\lambda, \delta_{k} \in \mathbb{R}$,

$$
\lambda=\frac{3}{2} \mathbf{e}_{i} \mathbf{Q}_{i j} \mathbf{e}_{j}
$$

and

$$
\mathbf{Q}_{i j} \mathbf{e}_{j}=\frac{2}{3} \lambda \mathbf{e}_{i}-\delta_{1} \mathbf{f}_{i}-\delta_{2} \mathbf{g}_{i} .
$$


Then

$$
|\mathbf{Q}|^{2}=\frac{2}{3} \lambda^{2}+2 \sum_{k=1}^{4} \delta_{k}^{2} \geq \frac{3}{2}\left(\mathbf{Q}_{i j} \mathbf{e}_{j}\right)^{2}
$$

and

$$
\left(\mathbf{Q}_{i j} \mathbf{e}_{j}\right)^{2}=\frac{4 \lambda^{2}}{9}+\delta_{1}^{2}+\delta_{2}^{2} \geq\left(\mathbf{e}_{i} \mathbf{Q}_{i j} \mathbf{e}_{j}\right)^{2} .
$$

We note that we have equality above when $\delta_{k}=0$ for all $k=1 \ldots 4$.

Combining (35) and (36), we have the following expression for $G(\mathbf{Q})$ as shown below:

$G(\mathbf{Q})=\frac{2}{3} W_{11} \lambda+\frac{4}{9}\left[\frac{3}{2} W_{21}+W_{22}+W_{23}\right] \lambda^{2}+\left(2 W_{21}+W_{23}\right)\left(\delta_{1}^{2}+\delta_{2}^{2}\right)+2 W_{21}\left(\delta_{3}^{2}+\delta_{4}^{2}\right)$.

From (32), we have $2 W_{21}+W_{23}, W_{21}>0$ and therefore, the last two terms are non-negative in (37). Consider the function

$$
f(\lambda)=\frac{2}{3} W_{11} \lambda+\frac{4}{9}\left[\frac{3}{2} W_{21}+W_{22}+W_{23}\right] \lambda^{2} .
$$

The function $f$ has a well-defined minimum at $\lambda=-\frac{3 W_{11}}{4\left(\frac{3}{2} W_{21}+W_{22}+W_{23}\right)}$, since $\left(\frac{3}{2} W_{21}+W_{22}+W_{23}\right)>0$ from (32). Therefore,

$$
G(\mathbf{Q}) \geq f\left(-\frac{3 W_{11}}{4\left(\frac{3}{2} W_{21}+W_{22}+W_{23}\right)}\right)=-\frac{W_{11}^{2}}{4\left(\frac{3}{2} W_{21}+W_{22}+W_{23}\right)}
$$

and Proposition 3 now follows

Comment: If $W_{11}=0$, then $G(\mathbf{Q}) \geq 0$ for all $\mathbf{Q} \in \bar{S}$ and the minimum is attained at the isotropic state $\mathbf{Q}=0$.

From Proposition 3, $G(\mathbf{Q})$ attains its minimum when

$$
\left(\mathbf{e}_{i} \mathbf{Q}_{i j} \mathbf{e}_{j}\right)^{2}=\left(\mathbf{Q}_{i j} \mathbf{e}_{j}\right)^{2}=\frac{W_{11}^{2}}{4\left(\frac{3}{2} W_{21}+W_{22}+W_{23}\right)^{2}} .
$$

The corresponding minimizer is

$$
\mathbf{Q}_{i j}=-\frac{3}{4} \frac{W_{11}}{\left(\frac{3}{2} W_{21}+W_{22}+W_{23}\right)}\left(\mathbf{e}_{i} \mathbf{e}_{j}-\frac{1}{3} \delta_{i j}\right) .
$$

This is identical to equation $(A 4)$ in [11]. It follows that $\mathbf{e}$ is an eigenvector of the minimizer in (39) and the corresponding eigenvalue is

$$
\lambda_{\mathbf{e}}=-\frac{W_{11}}{2\left(\frac{3}{2} W_{21}+W_{22}+W_{23}\right)} .
$$

Therefore, if $W_{11}>0\left(\lambda_{e}<0\right)$, then the minimizer favours alignment in a plane perpendicular to e (homeotropic alignment) and if $W_{11}<0\left(\lambda_{e}>0\right)$, then the minimizer favours alignment parallel to e. 
Consider the following Landau-de Gennes energy functional:

$$
I_{L G}[\mathbf{Q}]=\int_{\Omega} \frac{L}{2}|\nabla \mathbf{Q}|^{2}+f_{B}(\mathbf{Q}) d V+\int_{\partial \Omega} G(\mathbf{Q}) d S
$$

where $G$ has been defined in (30). As before, we take the admissible Qtensors to belong to the Sobolev space $W^{1,2}(\Omega ; \bar{S})$. It is straightforward to compute the corresponding Euler-Lagrange equations as shown below:

$$
\begin{aligned}
& L \Delta \mathbf{Q}_{i j}=a^{2} \mathbf{Q}_{i j}-b^{2}\left(\mathbf{Q}_{i k} \mathbf{Q}_{k j}-\frac{\delta_{i j}}{3} \operatorname{tr}\left(\mathbf{Q}^{2}\right)\right)+c^{2} \mathbf{Q}_{i j} \operatorname{tr}\left(\mathbf{Q}^{2}\right) \quad \text { on } \Omega \\
& L \nu_{k} \frac{\partial \mathbf{Q}_{i j}}{\partial \mathbf{x}_{k}}+W_{11} \mathbf{e}_{i} \mathbf{e}_{j}+2 W_{21} \mathbf{Q}_{i j}+2 W_{22}\left(\mathbf{e}_{p} \mathbf{Q}_{p q} \mathbf{e}_{q}\right) \mathbf{e}_{i} \mathbf{e}_{j}+2 W_{23}\left(\mathbf{Q}_{i p} \mathbf{e}_{p}\right) \mathbf{e}_{j}- \\
& -\left(W_{11}+2\left(W_{22}+W_{23}\right) \mathbf{e}_{q} \mathbf{Q}_{p q} \mathbf{e}_{p}\right) \frac{\delta_{i j}}{3}=0 \quad \text { on } \partial \Omega
\end{aligned}
$$

where $\nu$ is the unit outward normal to $\partial \Omega$.

Proposition 4. Let $\mathbf{Q}$ be a smooth solution of the Euler-Lagrange equations (41). Then $\mathbf{Q}$ satisfies the following global upper bound on $\bar{\Omega}$ :

$|\mathbf{Q}|^{2}(\mathbf{x}) \leq \max \left\{\frac{2}{3} s_{+}^{2}, \frac{W_{11}^{2}}{8\left(\frac{3}{2} W_{21}+W_{22}+W_{23}\right)}\left(\frac{3}{\left(\frac{3}{2} W_{21}+W_{22}+W_{23}\right)}+\frac{1}{\min \left\{2 W_{21}, 2 W_{21}+W_{23}\right\}}\right)\right.$

where $s_{+}$has been defined in Proposition 1.

Proof: We consider two cases separately. First, we assume that the function $|\mathbf{Q}|^{2}: \Omega \rightarrow \mathbb{R}$ attains its global maximum at an interior point $\mathbf{x}^{*} \in \Omega$. Then we can repeat the same arguments as in Proposition 1 to conclude that

$$
|\mathbf{Q}|^{2}\left(\mathbf{x}^{*}\right) \leq \frac{2}{3} s_{+}^{2} .
$$

Secondly, we assume that the function $|\mathbf{Q}|^{2}: \Omega \rightarrow \mathbb{R}$ attains its maximum at a boundary point $\mathbf{x}_{0} \in \partial \Omega$. We multiply both sides of

$$
\begin{aligned}
& L \nu_{k} \frac{\partial \mathbf{Q}_{i j}}{\partial \mathbf{x}_{k}}+W_{11} \mathbf{e}_{i} \mathbf{e}_{j}+2 W_{21} \mathbf{Q}_{i j}+2 W_{22}\left(\mathbf{e}_{p} \mathbf{Q}_{p q} \mathbf{e}_{q}\right) \mathbf{e}_{i} \mathbf{e}_{j}+2 W_{23}\left(\mathbf{Q}_{i p} \mathbf{e}_{p}\right) \mathbf{e}_{j}- \\
& -\left(W_{11}+2\left(W_{22}+W_{23}\right) \mathbf{e}_{q} \mathbf{Q}_{p q} \mathbf{e}_{p}\right) \frac{\delta_{i j}}{3}=0
\end{aligned}
$$

by $\mathbf{Q}_{i j}$ to obtain

$\frac{L}{2} \nu_{k} \frac{\partial|\mathbf{Q}|^{2}}{\partial \mathbf{x}_{k}}+W_{11} \mathbf{e}_{i} \mathbf{Q}_{i j} \mathbf{e}_{j}+2 W_{21}|\mathbf{Q}|^{2}+2 W_{22}\left(\mathbf{e}_{p} \mathbf{Q}_{p q} \mathbf{e}_{q}\right)^{2}+2 W_{23}\left(\mathbf{Q}_{i p} \mathbf{e}_{p}\right)^{2}=0$.

Since $|\mathbf{Q}|^{2}$ attains its global maximum at $\mathbf{x}_{0} \in \partial \Omega$, we have that

$$
\nu_{k} \frac{\partial|\mathbf{Q}|^{2}}{\partial \mathbf{x}_{k}}\left(\mathbf{x}_{0}\right) \geq 0
$$


Therefore, we must have

$$
W_{11} \mathbf{e}_{i} \mathbf{Q}_{i j} \mathbf{e}_{j}+2 W_{21}|\mathbf{Q}|^{2}+2 W_{22}\left(\mathbf{e}_{p} \mathbf{Q}_{p q} \mathbf{e}_{q}\right)^{2}+2 W_{23}\left(\mathbf{Q}_{i p} \mathbf{e}_{p}\right)^{2} \leq 0 .
$$

Using (35) and (36), we obtain the following inequality in terms of $\lambda$ and the $\delta_{k}$ 's:

$$
\frac{2 W_{11}}{3} \lambda+\frac{8}{9} \lambda^{2}\left(\frac{3}{2} W_{21}+W_{22}+W_{23}\right)+2\left\{\left(2 W_{21}+W_{23}\right)\left(\delta_{1}^{2}+\delta_{2}^{2}\right)+2 W_{21}\left(\delta_{3}^{2}+\delta_{4}^{2}\right)\right\}
$$

where we have expressed $|\mathbf{Q}|^{2}$ and $\left(\mathbf{Q}_{i p} \mathbf{e}_{p}\right)^{2}$ in terms of $\lambda$ and the $\delta_{k}$ 's. Since $\left(2 W_{21}+W_{23}\right)>0$ and $W_{21}>0$ from the hypothesis (32), we must have

$$
\frac{2 W_{11}}{3} \lambda+\frac{8}{9} \lambda^{2}\left(\frac{3}{2} W_{21}+W_{22}+W_{23}\right) \leq 0
$$

or equivalently

$$
\lambda^{2} \leq \frac{9}{16} \frac{W_{11}^{2}}{\left(\frac{3}{2} W_{21}+W_{22}+W_{23}\right)^{2}} .
$$

Next, consider the function

$$
f(\lambda)=\frac{2 W_{11}}{3} \lambda+\frac{8}{9} \lambda^{2}\left(\frac{3}{2} W_{21}+W_{22}+W_{23}\right)
$$

which has a well-defined minimum at $\lambda=-\frac{3}{8} \frac{W_{11}}{\left(\frac{3}{2} W_{21}+W_{22}+W_{23}\right)}$. Therefore, we have the following sequence of inequalities

$$
\begin{aligned}
& -\frac{W_{11}^{2}}{8\left(\frac{3}{2} W_{21}+W_{22}+W_{23}\right)}+\min \left\{\left(2 W_{21}+W_{23}\right), 2 W_{21}\right\} 2 \sum_{k=1}^{4} \delta_{k}^{2} \leq \\
& \frac{2 W_{11}}{3} \lambda+\frac{8}{9} \lambda^{2}\left(\frac{3}{2} W_{21}+W_{22}+W_{23}\right)+2\left\{\left(2 W_{21}+W_{23}\right)\left(\delta_{1}^{2}+\delta_{2}\right)+2 W_{21}\left(\delta_{3}^{2}+\delta_{4}^{2}\right)\right\}
\end{aligned}
$$

It follows straightaway from (45) that

$$
2 \sum_{k=1}^{4} \delta_{k}^{2} \leq \frac{W_{11}^{2}}{8\left(\frac{3}{2} W_{21}+W_{22}+W_{23}\right)} \frac{1}{\min \left\{\left(2 W_{21}+W_{23}\right), 2 W_{21}\right\}}
$$

and combining (46), (44) and (35), we obtain

$$
|\mathbf{Q}|^{2}\left(\mathbf{x}_{0}\right) \leq \frac{W_{11}^{2}}{8\left(\frac{3}{2} W_{21}+W_{22}+W_{23}\right)}\left(\frac{3}{\left(\frac{3}{2} W_{21}+W_{22}+W_{23}\right)}+\frac{1}{\min \left\{2 W_{21}, 2 W_{21}+W_{23}\right\}}\right) .
$$

Finally, we use the interior upper bound in Proposition 1 and (42) follows. 
The arguments in Proposition 4 can be adapted to include the effects of an electric field:

$$
I_{L G}[\mathbf{Q}]=\int_{\Omega} \frac{L}{2}|\nabla \mathbf{Q}|^{2}+f_{B}(\mathbf{Q})+F(\mathbf{Q}, \mathbf{E}) d V+\int_{\partial \Omega} G(\mathbf{Q}) d S .
$$

Let $\mathbf{Q}$ be a local or global smooth minimizer of $I_{L G}$ defined above. Then

$$
\begin{aligned}
& |\mathbf{Q}|^{2} \leq \max \left\{F_{3}^{2},\right. \\
& \left.\max \left\{\frac{2}{3} s_{+}^{2}, \frac{W_{11}^{2}}{8\left(\frac{3}{2} W_{21}+W_{22}+W_{23}\right)}\left(\frac{3}{\left(\frac{3}{2} W_{21}+W_{22}+W_{23}\right)}+\frac{1}{\min \left\{2 W_{21}, 2 W_{21}+W_{23}\right\}}\right)\right\} \text { s }\right\}
\end{aligned}
$$

on $\bar{\Omega}$, where $F_{3}$ has been defined in Proposition 2 .

\section{Time-dependent problems}

In this section, we study the time-evolution of the norm of a $\mathbf{Q}$-tensor configuration as it approaches a steady-state solution. We first study the evolution of a Q-tensor configuration, under gradient flow of the associated Landau-de Gennes energy functional, with suitable initial conditions and fixed dirichlet boundary conditions. Consider the following initial, boundary-value problem $[16]$ :

$$
\begin{aligned}
& \gamma \frac{\partial \mathbf{Q}_{i j}}{\partial t}=L \Delta \mathbf{Q}_{i j}-a^{2} \mathbf{Q}_{i j}+b^{2}\left(\mathbf{Q}_{i k} \mathbf{Q}_{k j}-\frac{\delta_{i j}}{3} \operatorname{tr}\left(\mathbf{Q}^{2}\right)\right)-c^{2} \mathbf{Q}_{i j} \operatorname{tr}\left(\mathbf{Q}^{2}\right) \\
& \mathbf{Q}(\mathbf{x}, 0)=\mathbf{Q}_{0}(\mathbf{x}) \quad \mathbf{x} \in \bar{\Omega} \\
& \mathbf{Q}(\mathbf{x}, t)=\mathbf{Q}_{b}(\mathbf{x}) \quad \mathbf{x} \in \partial \Omega
\end{aligned}
$$

where $\gamma>0$ is a viscosity coefficient and the initial condition $\mathbf{Q}_{0}$ is sufficiently smooth. The initial condition $\mathbf{Q}_{0}$ and the boundary condition $\mathbf{Q}_{b} \in W^{1,2}(\Omega ; \bar{S})$ satisfy the inequalities $\left|\mathbf{Q}_{0}\right|(\mathbf{x}) \leq \frac{1}{\sqrt{6}} s_{+}$for $\mathbf{x} \in \bar{\Omega}$ and $\left|\mathbf{Q}_{b}\right|(\mathbf{x}) \leq \frac{1}{\sqrt{6}} s_{+}$for $\mathbf{x} \in \partial \Omega$. Using standard bootstrapping arguments in the theory of partial differential equations, we have that all solutions of the initial, boundary-value problem (50) are smooth on the domain $\bar{\Omega} \times\{t \in[0, T]\}$ [7]. Our first result in this section demonstrates that the global upper bound in Proposition 1 is preserved under gradient-flow of the associated Landaude Gennes energy functional.

Proposition 5. Let $\mathbf{Q}(\mathbf{x}, t)$ be a smooth solution of the initial, boundaryvalue problem (50). Then

$$
|\mathbf{Q}|^{2}(\mathbf{x}, t) \leq \frac{2}{3} s_{+}^{2} \quad \mathbf{x} \in \bar{\Omega} ; t \in[0, T]
$$

for all $T>0$, where $s_{+}$has been defined in (6). 
Proof: We multiply both sides of the governing equation in (50) by $\mathbf{Q}_{i j}$ to obtain:

$$
\frac{\gamma}{2} \frac{\partial|\mathbf{Q}|^{2}}{\partial t}-\frac{L}{2} \Delta\left(|\mathbf{Q}|^{2}\right)=-L|\nabla \mathbf{Q}|^{2}-a^{2}|\mathbf{Q}|^{2}+b^{2} \operatorname{tr} \mathbf{Q}^{3}-c^{2}|\mathbf{Q}|^{4} .
$$

The function $|\mathbf{Q}|^{2}: \bar{\Omega} \times[0, T] \rightarrow \mathbb{R}$ is a smooth function of $\mathbf{x}$ and $t$. We first assume that $|\mathbf{Q}|^{2}$ attains its maximum at an interior point $\left(\mathbf{x}_{0}, t_{0}\right)$ where $0<t_{0}<T$ and that $|\mathbf{Q}|^{2}\left(\mathbf{x}_{0}, t_{0}\right)>\frac{2}{3} s_{+}^{2}$. Then

$$
\frac{\partial|\mathbf{Q}|^{2}}{\partial t}=0 \text { at }\left(\mathbf{x}_{0}, t_{0}\right)
$$

and

$$
g(|\mathbf{Q}|)=-a^{2}|\mathbf{Q}|^{2}+b^{2} \operatorname{tr} \mathbf{Q}^{3}-c^{2}|\mathbf{Q}|^{4}<0 \text { at }\left(\mathbf{x}_{0}, t_{0}\right)
$$

from the computations in (13) and (14), since $|\mathbf{Q}|^{2}\left(\mathbf{x}_{0}, t_{0}\right)>\frac{2}{3} s_{+}^{2}$ from the hypothesis. This implies that

$$
\frac{L}{2} \Delta\left(|\mathbf{Q}|^{2}\right)>0
$$

at $\left(\mathbf{x}_{0}, t_{0}\right)$ (from $\left.(52)\right)$, contradicting the definition of a maximum point in (22) [7].

Next, we suppose that $|\mathbf{Q}|^{2}$ attains its maximum at the boundary point $\left(\mathbf{x}_{0}, T\right)$, where $\mathbf{x}_{0} \in \Omega$ and $|\mathbf{Q}|^{2}\left(\mathbf{x}_{0}, t_{0}\right)>\frac{2}{3} s_{+}^{2}$. Then

$$
\frac{\partial|\mathbf{Q}|^{2}}{\partial t} \geq 0 \text { at }\left(\mathbf{x}_{0}, T\right)
$$

and $g\left(|\mathbf{Q}|\left(\mathbf{x}_{0}, T\right)\right)<0$, where $g$ has been defined in (54). This then necessarily implies that

$$
\frac{L}{2} \Delta\left(|\mathbf{Q}|^{2}\right)>0
$$

at $\left(\mathbf{x}_{0}, T\right)$, contradicting the definition of a maximum point as above. Finally, if $\mathbf{x}_{0} \in \partial \Omega$, then $|\mathbf{Q}|^{2} \leq \frac{1}{6} s_{+}^{2}$ on $\bar{\Omega} \times[0, T]$, by virtue of our hypothesis on the fixed boundary condition $\mathbf{Q}_{b}$ in (50). Proposition 5 now follows.

Next, we consider the related problem of time-evolution of $\mathbf{Q}$-tensor configurations in the presence of a constant electric field $\mathbf{E}$, with suitable initial and fixed dirichlet boundary conditions.

$$
\begin{aligned}
& \gamma \frac{\partial \mathbf{Q}_{i j}}{\partial t}=L \Delta \mathbf{Q}_{i j}-a^{2} \mathbf{Q}_{i j}+b^{2}\left(\mathbf{Q}_{i k} \mathbf{Q}_{k j}-\frac{\delta_{i j}}{3} \operatorname{tr}\left(\mathbf{Q}^{2}\right)\right)-c^{2} \mathbf{Q}_{i j} \operatorname{tr}\left(\mathbf{Q}^{2}\right)+\frac{1}{2} \epsilon_{0} \Delta \epsilon\left(\mathbf{E}_{i} \mathbf{E}_{j}-\frac{|\mathbf{E}|^{2}}{3} \delta_{i j}\right) \\
& \mathbf{Q}(\mathbf{x}, 0)=\mathbf{Q}_{0}(\mathbf{x}) \quad \mathbf{x} \in \bar{\Omega} \\
& \mathbf{Q}(\mathbf{x}, t)=\mathbf{Q}_{b}(\mathbf{x}) \quad \mathbf{x} \in \partial \Omega
\end{aligned}
$$

where $i, j=1 \ldots 3$, the initial condition $\mathbf{Q}_{0}$ is sufficiently smooth and we have the following inequalities: $\mathbf{Q}_{b} \in W^{1,2}(\Omega ; \bar{S})$ satisfies $\left|\mathbf{Q}_{b}\right|(\mathbf{x}) \leq$ $\frac{1}{2} F_{3}\left(a^{2}, b^{2}, c^{2}, \triangle \epsilon,|\mathbf{E}|^{2}\right)$ for $\mathbf{x} \in \partial \Omega$ and $\left|\mathbf{Q}_{0}\right|(\mathbf{x}) \leq \frac{1}{2} F_{3}\left(a^{2}, b^{2}, c^{2}, \triangle \epsilon,|\mathbf{E}|^{2}\right)$ for $\mathbf{x} \in \bar{\Omega}$ where $F_{3}$ has been defined in Proposition 2 . 
Proposition 6. Let $\mathbf{Q}(\mathbf{x}, t)$ be a smooth solution of the initial, boundaryvalue problem (55). Then

$$
|\mathbf{Q}|(\mathbf{x}, t) \leq F_{3}\left(a^{2}, b^{2}, c^{2}, \triangle \epsilon,|\mathbf{E}|^{2}\right) \quad \mathbf{x} \in \bar{\Omega} ; t \in[0, T]
$$

for all $T>0$, where $F_{3}$ has been defined in Proposition 2.

Proof: The proof of Proposition 6 is identical to the proof of Proposition 5 . We multiply both sides of the governing equation in (55) by $\mathbf{Q}_{i j}$ to obtain:

$\frac{\gamma}{2} \frac{\partial|\mathbf{Q}|^{2}}{\partial t}-\frac{L}{2} \Delta\left(|\mathbf{Q}|^{2}\right)=-L|\nabla \mathbf{Q}|^{2}-a^{2}|\mathbf{Q}|^{2}+b^{2} \operatorname{tr} \mathbf{Q}^{3}-c^{2}|\mathbf{Q}|^{4}+\frac{1}{2} \epsilon_{0} \triangle \epsilon \mathbf{E}_{i} \mathbf{Q}_{i j} \mathbf{E}_{j}$

Repeating the same arguments as above, we obtain the global upper bound (56) for the norm of any smooth solution $\mathbf{Q}(\mathbf{x}, t)$ of the initial, boundaryvalue problem (55), where $F_{3}$ is the largest positive zero of the cubic polynomial in (19). We note that if all zeros of the cubic polynomial in (19) are non-positive, then $|\mathbf{Q}|(\mathbf{x}, t) \leq \max \left\{\left\|\mathbf{Q}_{0}\right\|_{L^{\infty}(\bar{\Omega})},\left\|\mathbf{Q}_{b}\right\|_{L^{\infty}(\partial \bar{\Omega})}\right\}$ where $\|\mathbf{Q}\|_{L^{\infty}(\bar{\Omega})}=\operatorname{ess} \sup _{\mathbf{x} \in \bar{\Omega}}|\mathbf{Q}(\mathbf{x})|[7]$.

Finally, we consider the analogue of Proposition 4 in a dynamic setting. We work within a simple dissipation principle which requires that the Landau-de Gennes energy is a decreasing function of time [16] i.e.

$$
\frac{d I_{L G}}{d t}[\mathbf{Q}(\mathbf{x}, t)] \leq 0 \text {. }
$$

Then using the general form of the surface energy density $G$ in (30), we postulate that the governing equations are:

$$
\begin{aligned}
& \gamma \frac{\partial \mathbf{Q}_{i j}}{\partial t}=L \Delta \mathbf{Q}_{i j}-a^{2} \mathbf{Q}_{i j}+b^{2}\left(\mathbf{Q}_{i k} \mathbf{Q}_{k j}-\frac{\delta_{i j}}{3} \operatorname{tr}\left(\mathbf{Q}^{2}\right)\right)-c^{2} \mathbf{Q}_{i j} \operatorname{tr}\left(\mathbf{Q}^{2}\right) \quad \text { in } \Omega \\
& \gamma_{s} \frac{\partial \mathbf{Q}_{i j}}{\partial t}= \\
& =-\left\{L \nu_{k} \frac{\partial \mathbf{Q}_{i j}}{\partial \mathbf{x}_{k}}+W_{11} \mathbf{e}_{i} \mathbf{e}_{j}+2 W_{21} \mathbf{Q}_{i j}+2 W_{22}\left(\mathbf{e}_{p} \mathbf{Q}_{p q} \mathbf{e}_{q}\right) \mathbf{e}_{i} \mathbf{e}_{j}+2 W_{23}\left(\mathbf{Q}_{i p} \mathbf{e}_{p}\right) \mathbf{e}_{j}-\alpha \frac{\delta_{i j}}{3}\right\} \text { on } \partial \Omega \\
& \mathbf{Q}(\mathbf{x}, 0)=\mathbf{Q}_{0}(\mathbf{x}) \quad \mathbf{x} \in \bar{\Omega}
\end{aligned}
$$

where $\gamma, \gamma_{s}$ are bulk and surface viscosity coefficients respectively and $\alpha\left(\mathbf{Q}, W_{11}, W_{21}, W_{22}, W_{23}\right)=$ $\left(W_{11}+2\left(W_{22}+W_{23}\right) \mathbf{e}_{q} \mathbf{Q}_{p q} \mathbf{e}_{p}\right)$.

As before, we multiply both sides of the governing equations in (58) by $\mathbf{Q}_{i j}$ to obtain the following:

$$
\begin{aligned}
& \frac{\gamma}{2} \frac{\partial|\mathbf{Q}|^{2}}{\partial t}-\frac{L}{2} \Delta|\mathbf{Q}|^{2}=-L|\nabla \mathbf{Q}|^{2}-a^{2}|\mathbf{Q}|^{2}+b^{2} \operatorname{tr} \mathbf{Q}^{3}-c^{2}|\mathbf{Q}|^{4} \quad \text { in } \Omega \\
& \frac{\gamma_{s}}{2} \frac{\partial|\mathbf{Q}|^{2}}{\partial t}= \\
& -\left\{\frac{L}{2} \nu_{k} \frac{\partial|\mathbf{Q}|^{2}}{\partial \mathbf{x}_{k}}+W_{11} \mathbf{e}_{i} \mathbf{Q}_{i j} \mathbf{e}_{j}+2 W_{21}|\mathbf{Q}|^{2}+2 W_{22}\left(\mathbf{e}_{p} \mathbf{Q}_{p q} \mathbf{e}_{q}\right)^{2}+2 W_{23}\left(\mathbf{Q}_{i p} \mathbf{e}_{p}\right)^{2}\right\}
\end{aligned}
$$


Let $\mathbf{Q}(\mathbf{x}, t)$ be a smooth solution of the problem (58). If $|\mathbf{Q}|^{2}: \bar{\Omega} \times[0, T] \rightarrow \mathbb{R}$ attains its maximum at an interior point $\left(\mathbf{x}_{0}, t_{0}\right)$, where $\mathbf{x}_{0} \in \Omega$ and $0<t_{0} \leq$ $T$, then we can repeat the same arguments as in Proposition 5 to deduce that

$$
|\mathbf{Q}|^{2}\left(\mathbf{x}_{0}, t_{0}\right) \leq \frac{2}{3} s_{+}^{2}
$$

where $s_{+}$has been defined in (6). If $|\mathbf{Q}|^{2}: \bar{\Omega} \times[0, T] \rightarrow \mathbb{R}$ attains it maximum at $\left(\mathbf{x}_{0}, t_{0}\right)$ where $\mathbf{x}_{0} \in \partial \Omega$ and $0<t_{0} \leq T$, then

$$
\frac{\partial|\mathbf{Q}|^{2}}{\partial t} \geq 0
$$

and

$$
\nu_{k} \frac{\partial|\mathbf{Q}|^{2}}{\partial \mathbf{x}_{k}} \geq 0
$$

at $\left(\mathbf{x}_{0}, t_{0}\right)$. On the other hand, we can repeat the calculations in Proposition 4 to deduce that

$$
W_{11} \mathbf{e}_{i} \mathbf{Q}_{i j} \mathbf{e}_{j}+2 W_{21}|\mathbf{Q}|^{2}+2 W_{22}\left(\mathbf{e}_{p} \mathbf{Q}_{p q} \mathbf{e}_{q}\right)^{2}+2 W_{23}\left(\mathbf{Q}_{i p} \mathbf{e}_{p}\right)^{2}>0
$$

for

$$
|\mathbf{Q}|^{2}>\frac{W_{11}^{2}}{8\left(\frac{3}{2} W_{21}+W_{22}+W_{23}\right)}\left(\frac{3}{\left(\frac{3}{2} W_{21}+W_{22}+W_{23}\right)}+\frac{1}{\min \left\{2 W_{21}, 2 W_{21}+W_{23}\right\}}\right) .
$$

Therefore, for (60) to hold on the boundary, we must have

$$
|\mathbf{Q}|^{2}(\mathbf{x}, t) \leq \max \left\{\frac{2}{3} s_{+}^{2}, \beta\left(W_{11}, W_{21}, W_{22}, W_{23}\right)\right\} \quad(\mathbf{x}, t) \in \bar{\Omega} \times[0, T]
$$

where

$\beta\left(W_{11}, W_{21}, W_{22}, W_{23}\right)=\frac{W_{11}^{2}}{8\left(\frac{3}{2} W_{21}+W_{22}+W_{23}\right)}\left(\frac{3}{\left(\frac{3}{2} W_{21}+W_{22}+W_{23}\right)}+\frac{1}{\min \left\{2 W_{21}, 2 W_{21}+W_{23}\right\}}\right.$

These calculations for the problem (58) can be easily adapted to include the effects of an electric energy density $F(\mathbf{Q}, \mathbf{E})$, as in (15). The corresponding result is

$|\mathbf{Q}|^{2}(\mathbf{x}, t) \leq \max \left\{F_{3}^{2}\left(a^{2}, b^{2}, c^{2}, \triangle \epsilon,|\mathbf{E}|^{2}\right), \beta\left(W_{11}, W_{21}, W_{22}, W_{23}\right)\right\} \quad(\mathbf{x}, t) \in \bar{\Omega} \times[0, T]$

where $F_{3}$ is the largest positive zero of the cubic polynomial in (19). 


\section{Three-constant elastic energy density}

The results in Sections 2 and 3 focus on the one-constant elastic energy density, $\frac{L}{2}|\nabla \mathbf{Q}|^{2}$, where $L>0$ is a material-dependent elastic constant. In this section, we consider a more general elastic energy density $w(\nabla \mathbf{Q})$, such as the one proposed in $[16,5]$ :

$$
w(\nabla \mathbf{Q})=\frac{1}{2}\left(L_{1}|\nabla \mathbf{Q}|^{2}+L_{2} \mathbf{Q}_{i j, j} \mathbf{Q}_{i k, k}+L_{3} \mathbf{Q}_{i j, k} \mathbf{Q}_{i k, j}\right) \quad i, j, k=1 \ldots 3
$$

where the elastic constants are constrained as shown below

$$
L_{1}>0,-L_{1}<L_{3}<2 L_{1},-\frac{3}{5} L_{1}-\frac{1}{10} L_{3}<L_{2} .
$$

The elastic energy density $w(\nabla \mathbf{Q})$ in (64) is quadratic in $\nabla \mathbf{Q}$ and the constraints on the $L_{i}$ 's in (65) guarantee the following coerciveness property $[5]$

$$
w(\nabla \mathbf{Q}) \geq \alpha|\nabla \mathbf{Q}|^{2}, \alpha>0
$$

for all $\mathbf{Q} \in W^{1,2}(\Omega ; \bar{S})$.

Consider the following Landau-de Gennes energy functional:

$$
I_{L G}[\mathbf{Q}]=\int_{\Omega} w(\nabla \mathbf{Q})+f_{B}(\mathbf{Q}) d V
$$

where $w(\nabla \mathbf{Q})$ has been defined in (64) and $f_{B}$ has been defined in (4). We define our admissible space to be $\mathcal{A}=\left\{\mathbf{Q} \in W^{1,2}(\Omega ; \bar{S}) ; \mathbf{Q}=\mathbf{Q}_{b}\right.$ on $\left.\partial \Omega\right\}$ where $\mathbf{Q}_{b} \in W^{1,2}(\Omega ; \bar{S})$ is smooth by assumption. The admissible space $\mathcal{A}$ is non-empty (the boundary condition $\mathbf{Q}_{b} \in \mathcal{A}$ ). The energy functional $I_{L G}$ in $(67)$ is bounded from below, since $w(\nabla \mathbf{Q})$ obeys the coerciveness estimate in (66). Finally, $w(\nabla \mathbf{Q})$ is quadratic and hence, convex in $\nabla \mathbf{Q}$. Therefore, $I_{L G}$ in (67) is weakly lower semicontinuous on our admissible space and hence, we are guaranteed the existence of a global $I_{L G}$-minimizer in our admissible space $[7,4,5]$.

The corresponding Euler-Lagrange equations are

$$
\frac{\partial}{\partial \mathbf{x}_{k}}\left(\frac{\partial w(\nabla \mathbf{Q})}{\partial \mathbf{Q}_{i j, k}}\right)=\frac{\partial f_{B}}{\partial \mathbf{Q}_{i j}}+\lambda \delta_{i j}
$$

where $\mathbf{Q}_{i j, k}=\frac{\partial \mathbf{Q}_{i j}}{\partial \mathbf{x}_{k}}, \lambda$ is a Lagrange multiplier accounting for the tracelessness constraint and $\delta_{i j}$ is the Kronecker-delta symbol.

We briefly note that

$$
\frac{\partial \mathbf{Q}_{i j, j} \mathbf{Q}_{i k, k}}{\partial \mathbf{Q}_{i j, k}}=2 \delta_{j, k} \mathbf{Q}_{i \gamma, \gamma},
$$

and

$$
\frac{\partial \mathbf{Q}_{i j, k} \mathbf{Q}_{i k, j}}{\partial \mathbf{Q}_{i j, k}}=2 \mathbf{Q}_{i k, j}
$$


Substituting the above into (68), we obtain the following explicit form of the Euler-Lagrange equations :

$$
\begin{aligned}
& L_{1} \mathbf{Q}_{i j, k k}+L_{2}\left[\mathbf{Q}_{i \gamma, \gamma j}-\frac{1}{3} \mathbf{Q}_{\beta \gamma, \gamma \beta} \delta_{i j}\right]+L_{3}\left[\mathbf{Q}_{i k, j k}-\frac{1}{3} \mathbf{Q}_{\beta k, \beta k} \delta_{i j}\right]= \\
& =a^{2} \mathbf{Q}_{i j}-b^{2}\left(\mathbf{Q}_{i p} \mathbf{Q}_{p j}-\frac{1}{3} \operatorname{tr} \mathbf{Q}^{2} \delta_{i j}\right)+c^{2}\left(\operatorname{tr} \mathbf{Q}^{2}\right) \mathbf{Q}_{i j}
\end{aligned}
$$

where we have used the explicit form of $f_{B}$ in (4) and the formula $3 \lambda=$ $\delta_{i j}\left(\frac{\partial}{\partial \mathbf{x}_{k}}\left(\frac{\partial w(\nabla \mathbf{Q})}{\partial \mathbf{Q}_{i j, k}}\right)-\frac{\partial f_{B}}{\partial \mathbf{Q}_{i j}}\right)$.

As in the proof of Proposition 1, we multiply both sides of (69) by $\mathbf{Q}_{i j}$ to obtain

$L_{1} \frac{\partial}{\partial \mathbf{x}_{k}}\left[\mathbf{Q}_{i j} \mathbf{Q}_{i j, k}\right]+L_{2} \frac{\partial}{\partial \mathbf{x}_{j}}\left[\mathbf{Q}_{i j} \mathbf{Q}_{i \gamma, \gamma}\right]+L_{3} \frac{\partial}{\partial \mathbf{x}_{k}}\left[\mathbf{Q}_{i j} \mathbf{Q}_{i k, j}\right]=a^{2} \operatorname{tr} \mathbf{Q}^{2}-b^{2} \operatorname{tr} \mathbf{Q}^{3}+c^{2}\left(\operatorname{tr} \mathbf{Q}^{2}\right)^{2}+2 w(\nabla \mathbf{Q})$.

The techniques of Proposition 1 cannot be applied to (70) since the operator

$$
L_{1} \frac{\partial}{\partial \mathbf{x}_{k}}\left[\mathbf{Q}_{i j} \mathbf{Q}_{i j, k}\right]+L_{2} \frac{\partial}{\partial \mathbf{x}_{j}}\left[\mathbf{Q}_{i j} \mathbf{Q}_{i \gamma, \gamma}\right]+L_{3} \frac{\partial}{\partial \mathbf{x}_{k}}\left[\mathbf{Q}_{i j} \mathbf{Q}_{i k, j}\right]
$$

cannot be interpreted as an elliptic operator acting on $|\mathbf{Q}|^{2}$. Hence, we cannot readily apply maximum principle type of arguments to the scalar equation (70) that yield a global upper bound for the norm of arbitrary solutions of the system (69).

\section{Discussion}

This paper focuses on the qualitative properties of liquid crystal configurations in confined three-dimensional geometries, subject to external electric fields and surface anchoring energies. We consider static equilibria and the time-evolution of these liquid crystalline systems, subject to suitable initial and boundary conditions. The governing equations are computed in each case and we obtain explicit bounds for the norm of the corresponding solutions in terms of the temperature, material-dependent thermotropic constants, external field parameters and surface anchoring coefficients. We emphasize that these bounds apply to all local and global minimizers of the corresponding Landau-de Gennes energy functional. Once we have an explicit global upper bound for the norm i.e.

$$
|\mathbf{Q}| \leq \Sigma_{0}
$$

for some explicit $\Sigma_{0}>0$, the corresponding order parameters $(S, R)$ in (1) are constrained to take values inside the following fundamental domain:

$$
T_{L G}=\left\{(S, R) \in \mathbb{R}^{2} ; 0 \leq R \leq \frac{S}{3} \text { and } 0 \leq R+\frac{S}{3} \leq \frac{\Sigma_{0}}{\sqrt{2}} \cdot\right\}
$$


As mentioned in Section 1, the Q-tensor order parameter is defined to be the normalized second moment of a probability distribution function within mean-field theoretical approaches. The corresponding fundamental domain $T_{M F}$ in the $(S, R)$-plane is

$$
T_{M F}=\left\{(S, R) \in \mathbb{R}^{2} ; 0 \leq R \leq \frac{S}{3} \text { and } 0 \leq R+\frac{S}{3} \leq \frac{2}{3} .\right\}
$$

We can systematically compare the continuum domain $T_{L G}$ with the meanfield domain $T_{M F}$ to delineate the parameter regimes where the Landaude Gennes predictions match the mean-field predictions and the parameter regimes where the two theoretical approaches are inconsistent. The Landaude Gennes theory makes physically unrealistic predictions inconsistent with (72) for low temperatures, strong electric fields and large surface anchoring coefficients and this has been demonstrated for the simple set-up in Proposition 1 , in [14].

We briefly comment on Proposition 2. The global upper bound in (21) is not optimal in the sense that it only depends on the strength of the applied electric field and not on its direction. As an example, consider minimizers of the Landau-de Gennes energy functional (16) in the limit $L \rightarrow 0^{+}$. This can be viewed as a problem in singular perturbation theory. As $L \rightarrow 0^{+}$, the minimizers will converge (in some suitable norm) to minimizers of the augmented bulk energy density

$$
f_{B, \mathbf{E}}(\mathbf{Q})=f_{B}(\mathbf{Q})+F(\mathbf{Q}, \mathbf{E})
$$

where $f_{B}$ and $F$ have been defined in (4) and (15) respectively (see [3] for a similar problem). These minimizers will typically exhibit boundary layers near the confining surfaces depending on the interplay between the electric field and the imposed boundary conditions. The bound in (16) does not reflect this strong coupling between the electric field and the eigenvectors of the $\mathbf{Q}$-tensor solutions and hence, does not fully capture the physical scenarios.

In Proposition 4, we study a general surface energy density proposed in [11]. This surface energy density is the sum of four different components three of which are related to the preferred directions of alignment on the boundary and one of which is related to the scalar order parameters on the boundary. In Proposition 3, we analyze conditions for the well-posedness of this surface energy density and our results are similar to those reported in [11] and in Proposition 4, we obtain an explicit bound for the norm of all solutions in terms of the temperature, thermotropic constants and surface anchoring coefficients. This result reflects the interplay between bulk and surface effects and in the limit $L \rightarrow 0^{+}$, we expect that global energy minimizers will respect the bulk upper bound (10) in the interior of $\Omega$ and the surface upper bound (47) near the boundary, accompanied by the 
creation of fine intermediate interfaces. This will be investigated in future work.

In Section 3, we demonstrate that the static bounds obtained in Propositions 1,2, 4 carry over to dynamic situations i.e. are preserved under gradient flow of the associated Landau-de Gennes energy functionals. This result is relevant for dynamic processes, such as switching processes in liquid crystal displays and the motion of accompanying defects. In Section 4, we outline the technical difficulties of the general elastic energy density in (64). In [2], we propose a new continuum energy functional

$$
E[\mathbf{Q}]=\int_{\Omega} \frac{L}{2}|\nabla \mathbf{Q}|^{2}+\psi_{B}(\mathbf{Q}) d V
$$

where the modified bulk energy density $\psi_{B}$ blows up or diverges whenever the scalar order parameters $(S, R)$ take values outside $T_{M F}$ defined in (72) (see [10] for a similar formulation). We can show that a global energy minimizer of $E[\mathbf{Q}]$, in some suitably defined admissible space, respects the mean-field constraints in (72) for all temperature regimes [2]. However, our current methods don't work when we replace the one-constant energy density $|\nabla \mathbf{Q}|^{2}$ by a general quadratic energy density, such as $w(\nabla \mathbf{Q})$ in (64). This may be related to the technical difficulties in Section 4 where we cannot readily apply maximum-principle type of arguments to the scalar equation (70).

In conclusion, the results in this paper follow from elegant and rigorous maximum-principle type of arguments for elliptic and parabolic partial differential equations applied to liquid crystal problems. These methodologies are inspired from the Ginzburg-Landau theory for superconductors [3], are transferable to other problems in materials science modelling and are nice examples of how mathematics can be used to prove and quantify physically intuitive results.

\section{Acknowledgments}

This publication is based on work supported by Award No. KUK-C1-013-04 , made by King Abdullah University of Science and Technology (KAUST) to the Oxford Centre for Collaborative Applied Mathematics. The author thanks Professor Epifanio Virga for suggesting some of the problems in Section 2.

\section{References}

[1] J. M. Ball, Graduate lecture course "Mathematical theories of liquid crystals", 2007. 
[2] J. Ball \& A. Majumdar, 2009 Nematic liquid crystals : from Maier-Saupe to a continuum theory. Accepted for publication in Molecular Crystals and Liquid Crystals, Proceedings of the ECLC 2009.

[3] F. Bethuel, H. Brezis and F.Hélein, Asymptotics for the minimization of a Ginzburg-Landau functional. Calc. Var. Partial Differential Equations 1, no. 2, 123-148 (1993).

[4] B. Dacorogna, Direct methods in the calculus of variations. Applied Mathematical Sciences, 78, Springer, 1989.

[5] T. Davis and E. Gartland, Finite element analysis of the Landau-de Gennes minimization problem for liquid crystals. SIAM Journal of $\mathrm{Nu}-$ merical Analysis, 35, 336-362 (1998).

[6] P. G. De Gennes, The physics of liquid crystals. Oxford, Clarendon Press, 1974.

[7] L. Evans, Partial Differential Equations. American Mathematical Society, Providence, 1998.

[8] M. G. Forest, Q. Wang and H. Zhou, Homogeneous pattern selection and director instabilities of nematic liquid crystal polymers induced by elongational flows. Physics of Fluids, 12, no. 3, 490-498 (2000).

[9] M. G. Forest, Q. Wang and H. Zhou, Exact banded patterns from a DoiMarruci-Greco model of nematic liquid crystal polymers.Physical Review E, 61, no. 6, 6655-6662 (2000).

[10] J. Katriel, G.F. Kventsel, G.R. Luckhurst and T.J. Sluckin, Free Energies in the Landau and Molecular Field Approaches. Liquid Crystals 1, $337-55$ (1986).

[11] S.Krajl, R.Rosso \& E.Virga, Finite-size effects on order reconstruction around nematic defects. Physical Review E, 81, 1 (2010).

[12] F. H. Lin and C. Liu, Static and Dynamic Theories of Liquid Crystals. Journal of Partial Differential Equations, 14, no. 4, 289-330 (2001).

[13] A.Majumdar \& A.Zarnescu, The Landau-de Gennes theory of nematic liquid crystals: the Oseen-Frank limit and beyond. Archive of Rational Mechanics and Analysis, 196, No 1, 227-280 (2010).

[14] A. Majumdar, Equilibrium order parameters of liquid crystals in the Landau-de Gennes theory. European Journal of Applied Mathematics, 21, 181-203 (2010). 
[15] A.Majumdar, The Landau-de Gennes theory of nematic liquid crystals: Uniaxiality versus Biaxiality. Submitted to Communications in Mathematical Sciences.

[16] N. J. Mottram and C. Newton, Introduction to Q-tensor Theory. University of Strathclyde, Department of Mathematics, Research Report, 10 (2004).

[17] E. B. Priestley, P. J. Wojtowicz, and P. Sheng, Introduction to Liquid Crystals. Plenum, New York, 1975.

[18] M. J. Stephen and J. P. Straley, Physics of liquid crystals. Reviews of Modern Physics, 46, 617 - 701 (1974).

[19] M.R.Wilson, Progress in computer simulations of liquid crystals. International Reviews of Physical Chemistry, 24, 421 - 455 (2005). 



\section{RECENT REPORTS}

29/10 A Priori Error Estimates for Semidiscrete Finite Element Approximations to Equations of Motion Arising in Oldroyd Fluids of Or-

Goswami der One

30/10 The Landau-de Gennes theory of nematic liquid crystals: Uniaxiality versus Biaxiality

31/10 The Radial-Hedgehog Solution in Landau-de Gennes' theory

Pani

32/10 Nonlinear instability in flagellar dynamics: a novel modulation mechanism in sperm migration?

Majumdar

Majumdar

Gadelha

Gaffney

Smith

Kirkman-Brown

33/10 Error bounds on block GaussSeidel solutions of coupled multiphysics problem

Whiteley

Gillow

Tavener

Walter

34/10 A random projection method for sharp phase boundaries in lattice

Reis Boltzmann simulations

Dellar

35/10 Regularized Particle Filter with Langevin Resampling Step

Duan

Farmer

Moroz

36/10 Sequential Inverse Problems Bayesian Principles and the Logistic

Duan Map Example

Farmer

Moroz

37/10 Circumferential buckling instability of a growing cylindrical tube Moulton

Goriely

38/10 Preconditioners for state constrained optimal control problems

Stoll with Moreau-Yosida penalty function

Wathen

39/10 Local synaptic signaling enhances the stochastic transport of

Newby motor-driven cargo in neurons

Bressloff

40/10 Convection and Heat Transfer in Layered Sloping Warm-Water Aquifer

McKibbin

Hale

Style

Walters

41/10 Optimal Error Estimates of a Mixed Finite Element Method for

Goswami

Parabolic Integro-Differential Equations with Non Smooth Initial

Pani Data

Yadav

42/10 On the Linear Stability of the Fifth-Order WENO Discretization

Motamed

Macdonald

Ruuth 
43/10 Four Bugs on a Rectangle

Chapman

Lottes

Trefethen

44/10 Mud peeling and horizontal crack formation in drying clay

Style

Peppin

Cocks

45/10 Binocular Rivalry in a Competitive Neural Network with Synaptic

Kilpatrick Depression

Bressloff

46/10 A theory for the alignment of cortical feature maps during devel-

Bressloff opment

Oster

47/10 All-at-Once Solution if Time-Dependent PDE-Constrained Opti-

Stoll misation Problmes

Wathen

48/10 Possible role of differential growth in airway wall remodeling in asthma

Moulton

Goriely

49/10 Variational Data Assimilation Using Targetted Random Walks Cotter

Dashti

Robinson

Stuart

50/10 A model for the anisotropic response of fibrous soft tissues using

Flynn six discrete fibre bundles

Rubin

Nielsen

51/10 STOCHSIMGPU Parallel stochastic simulation for the Systems

Klingbeil Biology Toolbox 2 for MATLAB

Erban

Giles

Maini

Copies of these, and any other OCCAM reports can be obtained from:

Oxford Centre for Collaborative Applied Mathematics Mathematical Institute

24 - 29 St Giles'

Oxford

OX1 3LB

England

www.maths.ox.ac.uk/occam 